Rechtsmedizin 2009 · 19:389-390

DOI 10.1007/s00194-009-0637-9

Online publiziert: 15. November 2009

(c) Springer Medizin Verlag 2009

\author{
K. Püschel \\ Institut für Rechtsmedizin, Universitätsklinikum Hamburg-Eppendorf
}

\title{
Quo vadis „ärztliche Leichenschau“?
}

schauen müssen geahndet werden. Das Obduktionswesen muss erheblich umgestaltet werden. Nur Obduktionen bilden die Grundlage für: Qualitätskontrolle der ärztlichen Leichenschau, Qualitätskontrolle ärztlich-therapeutischer Maßnahmen, Aufklärung fremd verschuldeter Todesfälle, Aufklärung von Krankheiten und Todesursachen mit Relevanz für die Lebenden und die Verstorbenen. Die völlig unzureichende Sektionsfrequenz ist die Hauptursache für die derzeitige sehr kritische Situation" [1]. Im Grunde ist dies schon eine fast unendliche Geschichte ...

Nun hat die 78. Konferenz der Justizministerinnen und Justizminister im Juni 2007 in Berlin zur „Verbesserung der Qualität der äußeren Leichenschau" folgenden Beschluss gefasst: „In der Fachöffentlichkeit wird die Besorgnis geäußert, dass die derzeitige Praxis der Leichenschau die Feststellung nichtnatürlicher Todesfälle nicht immer sicher gewährleiste. Eine mögliche Ursache liegt darin, dass die Leichenschau nahezu in allen Ländern nicht durch entsprechend spezialisierte Ärztinnen und Ärzte durchgeführt werde. Die Justizministerinnen und Justizminister halten diese Thematik für klärungsbedürftig. Sie bitten ihre Vorsitzende, an das Innen- und Gesundheitsressort mit dem Ziel heranzutreten, das derzeitige System der Leichenschau zu überprüfen und gegebenenfalls gemeinsam ein Konzept zur Verbesserung der Leichenschau zu entwickeln."

Der Strafrechtsausschuss der Justizministerkonferenz hat daraufhin unter Beteiligung der Innenministerkonferenz, der Gesundheitsministerkonferenz und der Kultusministerkonferenz eine Projektgruppe konstituiert. Diese hat festgestellt, dass die Qualität der äußeren Leichenschau - wie die festgestellten Defizite zeigen - in Deutschland dringend der Verbesserung bedarf. Als wesentliche Voraussetzung hierfür wird die grundsätzliche Entkoppelung der Todesfeststellung von der Leichenschau angeführt. Hierbei bleibt jeder approbierte Arzt zur Vornahme der Todesfeststellung verpflichtet. Die nachfolgende äußere Leichenschau soll dagegen durch speziell im Rahmen einer Zusatzqualifikation fort- und weitergebildete Ärzte erfolgen und insoweit „professionalisiert “ werden. Die vorgesehene Honorierung dieser Maßnahmen soll angemessen verbessert werden.

Hierzu hat sich wiederum der Vorstand der Deutschen Gesellschaft für Rechtsmedizin kritisch geäußert und in einem Eckpunktepapier (im Juli 2009) Vorschläge zur Verbesserung der Leichenschau und zur Aufdeckung von nichtnatürlichen Todesfällen gemacht. Diese zielen auf eine weitere Verbesserung der Ausbildung im Medizinstudium, die postgraduale Fortbildung der Ärzte sowie auf eine als entscheidend dargestellte Steigerung der Obduktionsrate. Ohne Obduktion gibt es keinerlei Evaluation über die Qualität der Leichenschau. Nur die Obduktion führt zu einer eindeutigen Klärung. Allein aufgrund der (qualitativ noch so guten) äußeren Leichenschau kann bei unklarer Krankheitsvorgeschichte über die tatsächliche Todesursache allenfalls spekuliert werden. - Diese Position wird insbesondere durch die Beiträge von Rothschild und Große Perdekamp et al. in die- der Todesursache müssen eindeutig festgelegt werden. Grob fahrlässige Leichen- 
sem Schwerpunktthemenheft speziell vertieft. Gravierende - eigentlich kaum verständliche - Fehler bei der äußeren Leichenschau werden in den Falldarstellungen von Althaus u. Freislederer sowie Zweihoff u. Püschel dargestellt. Fieseler u. Peschel stellen den Aufbau des durch Ärzte des Instituts für Rechtsmedizin organisierten Leichenschaudienstes im Großraum München dar, der offenbar eine relevante Qualitätsverbesserung bedeutet. Seit Langem bekannte grundsätzliche Probleme, Unzulänglichkeiten und Fehler bei der Leichenschau sind unverkennbar. Die weiteren Beiträge von Madea sowie Dettmeyer u. Verhoff fassen alle medizinischen, kriminalistischen und rechtlichen Aspekte der Leichenschau einerseits sowie die strukturellen Probleme andererseits nochmals übersichtlich zusammen. Insgesamt gelingt hiermit eine überzeugende Standortbestimmung.

Einige eigene Gedanken, Ideen und Vorschläge, für die es derzeit sicherlich keine realen Ansätze der Umsetzung gibt, die aber als Zukunftsvision möglicherweise durchaus anregend sind, sollen hinzugefügt werden: Gemeint ist ein sehr viel weitergehendes Verständnis von der äußeren Leichenschau im Sinne einer umfassenden Qualitätssicherung in der Medizin, für die Sicherheit des Einzelnen und für die Rechtssicherheit im Allgemeinen. Selbst bei vergleichsweise banalen medizinischen Beschwerden ist es heutzutage selbstverständlich, dass die diesbezügliche Diagnostik mit einer sehr ausgefeilten Technik der Bildgebung erfolgt, z. B. Sonographie, Röntgen, Computertomographie (CT), Magnetresonanztomographie (MRT) usw. - Und was für eine „normale“, standardmäßige medizinische Diagnostik selbstverständlich ist, sollte doch auch eingesetzt werden, wenn die am weitesten gehende, einschneidendste und gravierendste Diagnose gestellt wird: nämlich „Tod“. Um die Todesursache genau festzustellen, sollten auch im Normalfall die ansonsten routinemäßig verfügbaren technischen Mittel und Laboruntersuchungen eingesetzt werden. Warum statten wir die hierfür zuständigen Ärzte (z. B. Rechtsmediziner) nicht so aus, dass auch im Rahmen einer Leichenschau routinemäßig eine CT- und eine MRT-Untersuchung durchgeführt werden? Und zu- sätzlich natürlich auch eine orientierende chemisch-toxikologische Analyse von Blut-, Urin- und Haarasservaten, die bei jedem Leichnam problemlos mit einem kleinen Eingriff gewonnen werden können. Dies wäre einem Staatswesen angemessen, das für das Leben aller Bürger einen hohen Qualitätsanspruch vertritt und in der Medizin sowie im Bereich der inneren Sicherheit und für das Recht ansonsten (fast) keine Kosten scheut.

\section{Korrespondenzadresse}

\section{Prof. Dr. K. Püschel}

Institut für Rechtsmedizin,

Universitätsklinikum Hamburg-Eppendorf

Butenfeld 34, 22529 Hamburg

pueschel@uke.de

Interessenkonflikt. Der korrespondierende Autor gibt an, dass kein Interessenkonflikt besteht

\section{Literatur}

1. Brinkmann B, Püschel K (1991) Definition natürlicher, unnatürlicher, unklarer Tod. Todesursachenklärung: Derzeitige Praxis. MedR 5(9):233-238

2. Brinkmann B, Du Chesne A (1993) Die Misere der ärztlichen Leichenschau in der Bundesrepublik Deutschland. Med Welt 44:697-701

3. Rückert S (2000) Tote haben keine Lobby. Die Dunkelziffer der vertuschten Morde. Hoffmann \& Campe, Hamburg

\section{Februar 2010}

Mainz 19.-20.02.2010

Kurs Verkehrsmed. Qualifikation

Themen: 16-h-Kurs zur Erlangung der verkehrsmed. Qualifikation

Auskunft: Frau C. Becker,

Akademie für Ärztl. Fortbildung,

Deutschhausplatz 3, 55116 Mainz,

Fon: 06131/2843813,

Fax: 06131/2843810,

becker@arztkolleg.de,

www.arztkolleg.de

Seattle (U.S.A.) 22.-27.02.2010

62nd AAFS Anniversary Meeting

Auskunft: American Academy

of Forensic Sciences

\section{August 2010}

Bonn 29.08.-02.09.2010

48th Annual Meeting of TIAFT

Joint Meeting with GTFCh

Themen: Alcohol, Drugs, and Driving,

Clinical Toxicology, Drug Monitoring,

Drugs of Abuse, Drug Facilitated Crimes

Auskunft: Herr Prof. Dr. Frank Musshoff,

TIAFT/GTFCh,

f.musshoff@uni-bonn.de,

http://www.gtfch.org/cms/images/stories/

tiaft2010_bonn_announcement.pdf

\section{September 2010}

Berlin 21.-25.09.2010

89. Jahrestagung

der Deutschen Gesellschaft

für Rechtsmedizin

Themen: Morphologie, Toxikologie,

Forensische Genetik, Didaktik

in der Rechtsmedizin

Auskunft: Frau Dr. Sarah Heinze,

Institut für Rechtsmedizin Berlin,

Fon: 030 901728135,

Fax: 030 901728154,

dgrm2010@charite.de,

http://www.rechtsmedizintagung2010. de/portal/

\section{Februar 2011}

Chicago (U.S.A.) 21.-26.02.2011

63rd AAFS Anniversary Meeting

Auskunft: American Academy

of Forensic Sciences

\section{September 2011}

Frankfurt/Main 26.09.-01.10.2011

90. Jahrestagung der DGRM

Auskunft: Herr Prof. Hansjürgen Bratzke, IRM Frankfurt 